\title{
The Mental Consulting System for the Prevention of Lifestyle-Related Diseases
}

\author{
Katsuyuki Nakano ${ }^{1}$; Hidetoshi Kashihara ${ }^{2}$; Masato Murakami ${ }^{3}$; Hisaki Haga ${ }^{1}$; Takaki Atobe ${ }^{1}$; Tatsuki Aiso ${ }^{1}$; \\ Migaku Matsuoka'; Yoshiharu Iwashita'; Masayuki Suda'; Keijiro Kiyoshima ${ }^{4}$; and Masaki Tamura ${ }^{2}$ \\ ${ }^{1}$ PL Comprehensive Research Institute, ${ }^{2}$ PL Tokyo Health Control Center, ${ }^{3}$ Nihon University Itabashi Hospital, and ${ }^{4}$ PL Osaka Health Check-up Center
}

\begin{abstract}
A mental consulting system to prevent lifestyle-related diseases was developed as an application of health risk appraisal (HRA). The self-administered questionnaire, known as the revised Mental General Questionnaire, Version 2 (MGQ2) was developed based on the results of statistical studies of the association between the incidences of lifestyle-related diseases and the results of a previously developed personality questionnaire. The MGQ2 was constructed using six personality scales related to cancer, diabetes mellitus, hypertension, cerebral apoplexy, heart diseases, and tooth lap diseases. The personality scales, of the above six diseases, consist of thirteen sub-scales. From the MGQ2 answers, scores and profiles of thirteen personality sub-scales were calculated and the appropriate mental advice was selected automatically for the test taker. High scores in the personality sub-scale questions indicate high-risk mental habits which can result in lifestyle-related diseases. The mental advice provided enables the test taker to take care of mental conditions in addition to the advice generally used to support physical conditions such as exercise and nutrition under development.
\end{abstract}

Key Words Mental Consultation; Lifestyle-Related Diseases; Prevention; Personality; Questionnaire

\section{INTRODUCTION}

The PL Tokyo Health Control Center and the PL Osaka Health Check-up Center were established in 1970 and 1971 to periodically study both the physical and mental (psychological) health of members from both centers. Both centers adopted a health checkup system examining the test takers twice a year.

For several years after the establishment of the above organizations, the individual physiological variations of the test takers' blood chemistry and their variances in sex, age and intra-individual and inter-individual variance were examined via the statistical analysis of the physiological data accumulated by the PL Tokyo Health Control Center. ${ }^{1)}{ }^{2)}$ As a result, we reported the significance of clinical evaluations based on subject-specific normal values in addition to those based on the population normal range of clinical tests. From this research, the importance of subject-spe-

Received: July 2, 2001.

1 Address; PL Comprehensive Research Institute, Church of Perfect Liberty, 2172-1 Shindo, Tondabayashi, Osaka, 584-8651 Japan. TEL; 81-721-23-6682; FAX; 81-721-23-3815, E-mail: katuyuki@po.cri. perfect-liberty.or.jp

2 Address; PL Tokyo Health Control Center, 16-1 Kamiyamacho, Shibuya-ku, Tokyo, 150-0047 Japan. TEL; 81-3-3469-1161.

Address; Nihon University Itabashi Hospital, 30-1, Kamimachi, Ooyaguchi, Itabashi-ku, Tokyo, 173-8610 Japan. TEL; 81-3-3972-8111.

4 Address; PL Osaka Health Check-up Center, Honmachi Eiwa Building, 4-2-10 Minamihonmachi, Chuoku, Osaka, 541-0054 Japan. TEL; 81-6-6241-0221.

* This paper is published by 11 authors and is a result of an eightyear joint research project which began in 1993 . cific normal ranges has been recognized and its clinical application on individual health monitoring has been expected.

The second step consisted of a statistical analysis predicting the onset of lifestyle-related diseases using the physiological data collected by the PL Tokyo Health Control Center performed after waiting for the accumulation of diagnostic information. Based on the results of these analyses, the computer program for the prediction of disease in ten years was developed to offer medical and nutritional advice by the method of health risk appraisal using clinical test data. ${ }^{3)}$

On the other hand, as a development of psychological or behavioral approach to health science and adult diseases, the coronaryprone personality or the type A behavior pattern in ischemic heart disease ${ }^{4)}$ and the cancer-prone personality or the type $\mathrm{C}$ behavior pattern in cancer ${ }^{5), 6)}$ have been examined as a characteristic personality or behavior pattern of those with these diseases. From this research, disease-prone personality types or behavior patterns, especially type A behavior, has been studied in various countries in the world, and the concept has been accepted by society. However, there are few applications ${ }^{7)}$ of mental health analysis using the personality scales as an effective health check-up methods at present.

The questionnaire data examining the respective personality types of people with lifestyle-related diseases such as diabetes mellitus and hypertension were collected in the database of the PL Tokyo Health Control Center. The questionnaire includes the general mental questionnaire, version 1 (MGQ1) and questionnaires examining the personality types of patients with diabetes, hypertension, heart disease and stroke. In 1981, a multivariate analysis of five-year risk factors of gastric cancer was performed using clinical tests and the MGQ1. ${ }^{8}$ For three years, beginning in 1993, case-control studies examining the personality types of patients with gastrointestinal cancer was performed at the PL Tokyo Health Control Center and the PL Hospital using a newly developed questionnaire. ${ }^{9) .10)}$ Furthermore, for four years beginning in 1996, a cohort study and case-control study of the personality of patients with diabetes and hypertension using the MGQ1 and other kinds of questionnaires had been performed. ${ }^{11-15)}$

Based on the results of the studies mentioned above, the MGQ2 (mental general questionnaire, version 2) ${ }^{16)}$ was developed to examine the respective personality types of people with lifestylerelated diseases such as cancer, diabetes, hypertension, heart disease, and stroke. Using the MGQ2, a mental consulting system for the prevention of lifestyle-related diseases was developed for health risk appraisal. ${ }^{17)}$

In this paper, we describe a revised MGQ2 and a new version of the "PL Mental Consulting System" using the MGQ2. We developed a computerized system to input the answers of the test takers, to calculate scores, to draw a profile of thirteen personality sub- 
scales, and to provide the appropriate mental advice needed for the prevention of lifestyle-related diseases.

\section{MATERIALS AND METHODS}

The database developed by the PL Tokyo Health Control Center included clinical test data and questionnaire data such from the

Table 1 The six personality scales and thirteen sub-scales of the revised MGQ2. Includes contents and meanings of the sub-scales.

\begin{tabular}{ll}
\hline Scales and sub-scales & Contents and meanings \\
\hline 1. Cancer related personality scales &
\end{tabular}

1-1 Dissatisfaction with disliked persons (6 items)

1-2 Feelings of persecution and helpless submission (7 items)

1-3 Feelings of obstinacy and stubbornness (6 items)

\footnotetext{
1-4 Feelings of justice and love of cleanliness (8 items)
}

An illogical person makes me unpleasant. People who pretend to be wise make me unpleasant. I do not like unnecessary interference. I think: "Hey, s/he did it again! How silly s/he is!" Once I begin to dislike someone I don't even want to hear his/her voice. A stubborn person makes me unpleasant.

I feel cornered by too much responsibility. I continuously feel burdened by my job and chores. I am mentally burdened while working. I feel mental agony when alone. I cannot trust others anymore. I regret and brood over my failures. I keep my feelings to myself because I think that I should not say anything.

Others say I am stubborn. I have to finish my own tasks so as to complete them according to my intentions even after I delegated them to others. I want to judge everything as being either black or white. I will do something no matter who or what is against me. I feel dissatisfied when an agreement isn't carried out exactly as planned. I cannot be flexible with my principles.

I become very unpleasant towards those who show favoritism. I do not allow others to behave in a dishonest manner. I am particular about trifling matters and think that things should be done my way. I have a strong sense of justice. I judge things as being either bad or good. I have a keen sense of responsibility. I can't be satisfied unless I have done something thoroughly. I can't count on anyone but myself.

1-5 Selfishness with foods and meals (4 items) I eat with feeling of anger. If there is only a mouthful of food left in my dish I won't eat it if I don't like it. I have specific tastes therefore I cook or season my own foods. When I eat my favorite foods, I don't feel satisfied unless I have eaten a lot.

2. Diabetes mellitus related personality scales

2-1 Dissatisfaction with irritating persons ( 7 items)

Whenever I see what others are doing, I feel that they should do it differently. I understand what others want to say before they complete their explanations and I become irritated. I am surprised when I meet a person whom I feel is ignorant. I become irritated when dealing with an ignorant person. I feel that others get in my way. I make quick decisions. I don't understand why others are laughing and what is so funny.

2-2 Favorite foods and meals (5 items)

I am willing to go to any length to get a particular food. I am sensitive to the taste of foods. I want to eat sweet foods carelessly. I put my favorite food aside and eat it last. I save and store favorite foods so that I can eat them when I want.

3. Hypertension related personality scales

3-1 Feelings of anxiety (7 items)

I think about my problems after I go to bed. I become anxious thinking about what I have to do or what may happen next. When I remember something unpleasant, I feel uneasy and am unable to let go. Once I ask others for something, I cannot help but remind them many times. Once I start to worry about something, I am unable to think about anything else. I have been told that I repeat myself. I think that I am an unlucky person.

3-2 Sturbbornness and dissatisfaction (4 items)

I hold my anger and I don't say anything when I am encounter people who have different ideas and opinions. I become disgusted with those who never change their attitude and sayings. I try not to say lies and not to make a mistake. I am poor at having a new hobby.

4. Cerebral apoplexy related personality scale

4-1 Anger and short-temper (8 items)

I feel bitter whenever I remember an unpleasant experience. I get upset and quit what I am doing. I put the blame on others and get angry. I want to tell others to quit making excuses and do as I say. I loose control over my words when I give way to my anger. I am short tempered. I am a hasty person. When others say something that upsets me I cannot let go.

5. Heart diseases related personality scales

5-1 Feelings of uneasiness (5 items)

I begin to worry whether my actions will cause negative repercussions in the future. I feel anxious for no particular reason. I tremble to think that I may make a mistake which will cause something irrecoverable. I become anxious if I feel that I could be criticized for something. I tend to think negatively.

5-2 Perseverance (4 items) I am afraid of bad results and hurry to take steps for an early solution. I try hard not to know fatigue. When I do it and can accomplish it, I am absorbed in it. I try hard to overcome problems which arise one after another.

6. Tooth lap diseases related personality scale

6-1 Complaints and patience

I want to question and to scold the failure and clumsiness of a person. I watch the behavior of a per-
son and I complain that this is "an already hopeless person". I am often told about a person's bad (4 items) behavior. I am mortified and grit my teeth. 
MGQ1 (160 questions) and personality tests for cancer (110 questions), diabetes mellitus (40 questions), hypertension (34 questions), cardiovascular diseases (136 questions), and heart diseases (44 questions). These personality questionnaires, excluding the data on cancer and cardiovascular diseases, were developed in 1969.

We studied the relationship between the results of these personality questionnaires and the incidences of the lifestyle-related diseases by epidemiological methods. A case-control study of the personality types of patients with gastrointestinal cancer including investigations regarding their spouses was also performed. ${ }^{9), 10)}$ The cohort studies including Cox proportional hazard model were performed using MGQ1 and its scales obtained by factor analysis, and case-control studies, using personality questionnaires of diabetes mellitus and hypertension for the analysis of personality of patients with these diseases. ${ }^{11)-15)}$

As a result of the cross sectional and longitudinal study, the factors and questions connected with these lifestyle-related diseases were found, and the second version of MGQ (called MGQ2 or PL mental test $)^{16)}$ was developed. Some questions in the MGQ2, especially questions related to cerebral apoplexy, heart disease and tooth lap disease have not been investigated because there was very few diagnostic data of patients with these diseases. Therefore, scales and questions related to diseases were selected based on the empirical knowledge gained from examinations by doctors and religious counsels of the Church of Perfect Liberty. The sentence questions were closely refined. Thus, a revised version of the self-administered questionnaire, known as MGQ2 was developed. The scales and questions of the MGQ2 were considered elements of personality scale as a mental background of lifestylerelated diseases.

The MGQ2 is composed of six personality scales related to cancer, diabetes mellitus, hypertension, cerebral apoplexy, heart diseases, and tooth lap diseases. The six personality scales related to the above diseases consist of a total of thirteen sub-scales.

\section{RESULTS}

As a result of the cross sectional and longitudinal studies on the association between the incidence of lifestyle-related diseases and several kinds of personality type questionnaires, factors and questions related to incidence of the diseases were selected from questions of the previous questionnaires. The revised MGQ2 consisting of 76 questions was developed as mentioned in Materials and methods.

The MGQ2 consists of six personality scales such as cancer, diabetes mellitus, hypertension, stroke, heart disease and tooth lap disease. Table 1 shows those six personality scales, and it also shows thirteen sub-scales of the MGQ2 related to above six diseases together with contents and meanings. Each of the six personality scales has sub-scales. The first personality scale (related to cancer) has five sub-scales. The second (diabetes) has two subscales. The third (hypertension) has two sub-scales. The fourth (cerebral apoplexy) has one sub-scale. The fifth (heart disease) has two sub-scales. The last (tooth lap disease) has one sub-scale. Furthermore, each sub-scale consists of the several numbers of question items shown in Table 1.

For example: Table 2 shows six question items included in "1-1. Dissatisfaction with disliked persons", first sub-scale of the cancer related personality scale. For example: question number 19 is "I know somebody who usually says and does illogical things, s/he makes me unpleasant". The square brackets on the right side in Table 2 indicate that this question or scale was selected based on the evidence that sex and age matched the odds ratio of this scale or question between cancer patients and normal controls was found to be significant by the above mentioned case-control study on the incidence of cancer. [CAQ Q47, S1] means that this question number 19 was adopted from the 47th question belonging to scale 1 of the personality questionnaire CAQ developed to examine cancer personality type.

Using the MGQ2, we developed the Internet web-based program for mental consultation to prevent lifestyle-related diseases. From the MGQ2 test takers' answers, scores and profiles of thirteen personality sub-scales were calculated and consulted with doctors. The appropriate mental advice was selected automatically for test takers. A high score in the personality sub-scales, implies mental behavior that puts the test taker at high risk for an incidence of lifestyle-related diseases. This computerized system is called the "PL mental consulting system." Utilizing the mental advice for health care, each test taker is able to take care of mental conditions in addition to receiving advice for physical conditions such as exercise and nutrition under development.

The top frame of the web page of the mental consulting system consists of an explanation of the system e.g.: "Your answers to the following 76 questions will enable the system to analyze your personality. Based on the results, you will be presented with advice for preventing lifestyle-related diseases." The link to the sample page, and input fields of the examination number, name, sex, member and age are continued.

Figure 1 shows the first 10 question items of "Part I" of the MGQ2 in the web page. A questionnaire with five multiple-choice questions was adopted, that is, the selection of answers:

0 . absolutely not, 1. rarely, 2. sometimes, 3. quite often, 4. always.

Although it is not shown in the Figure, from the 60th question belonging to "Part II" of the MGQ2, expression of answer is

Table 2 Question items included in first sub-scale of cancer related personality scales.

\begin{tabular}{ll}
\hline 1-1 Dissatisfaction with disliked persons (6 items) & \\
\hline 19. I know somebody who usually says and does illogical things, s/he makes me unpleasant. & [CAQ Q47, S1] \\
21. People who act as though they know it all upset me. & [CAQ Q61, S1] \\
36. I do not like unnecessary interference. & [CAQ Q58, S1] \\
45. Watching others' actions, I think: "Hey, s/he did it again! How silly s/he is!” & [CAQ Q62, S1] \\
49. Once I begin to dislike someone I don't even want to hear his/her voice. & [CAQ Q68, S1] \\
54. If a stubborn person doesn't listen to me, I become unpleasant. & [CAQ Q64, S1] \\
\hline
\end{tabular}

[ ] means that each question item was adopted from the question item of designated question number belonging to scale 1 of the personality questionnaire CAQ developed to examine cancer personality type. 


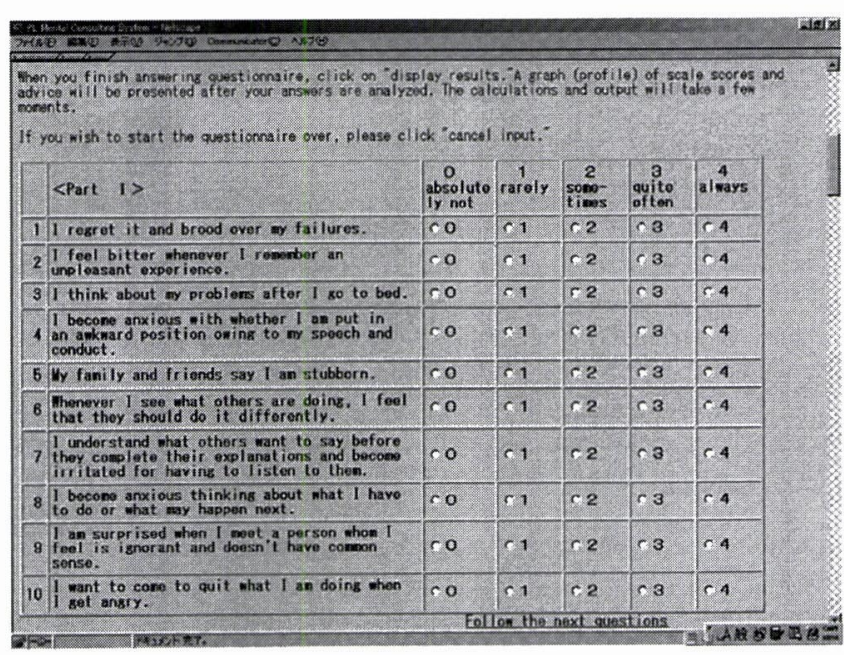

Fig. 1 The first ten question items of the revised MGQ2 on the PL mental consulting system web page.

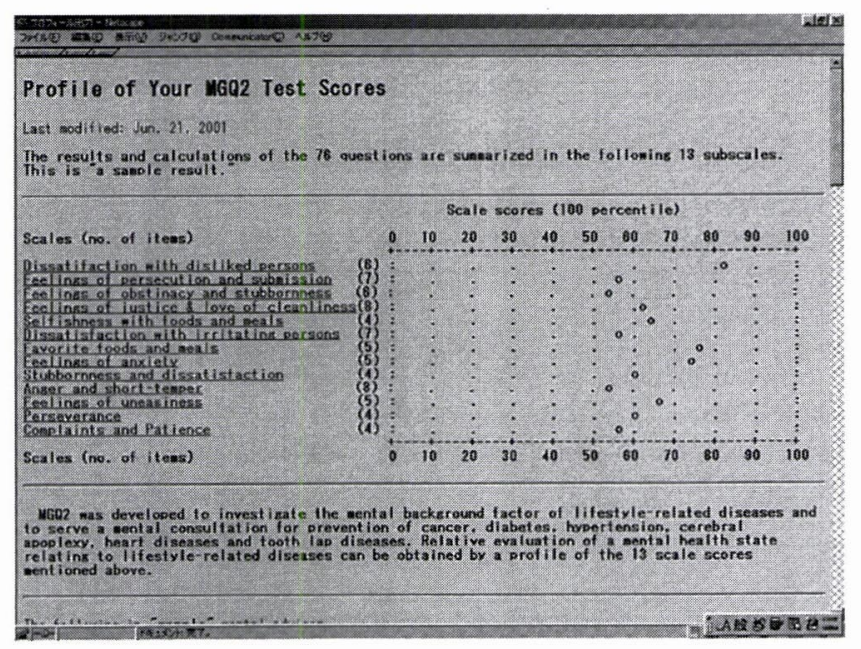

Fig. 2 A sample output of the profile results from thirteen personality sub-scales related to lifestyle-related diseases on the web page.

changed a little, that is, answers:

0 . absolutely not true, 1. a little bit true, 2. somewhat true, 3 . almost true, 4. absolutely true.

The number in front of an answer is used as item score. Scores of each sub-scale are calculated as a simple sum of item scores of questions belonging to the sub-scale.

Figure 2 is a sample output result of profiles thirteen personality sub-scales. This questionnaire's objective is the identification of personality types at high risk for getting cancer, diabetes, hypertension, cerebral apoplexy, heart diseases and tooth lap diseases. The relative evaluation of the mental health states associated with these lifestyle-related diseases can be obtained by a profile (or graph) of thirteen sub-scale scores as shown in this Figure. In this program, the explanation of how to read a result (profile) of the MGQ2 is shown as the table of name of thirteen sub-scales and those contents or meanings.

Finally, Fig. 3 shows sample advice generated by the sample result mentioned above. Messages of this sample contain a warning against the possible incidence of cancer, diabetes, and hypertension. The test taker is informed that the risks of these diseases will increase if $\mathrm{s} / \mathrm{he}$ continues to have such feelings and behaviors.

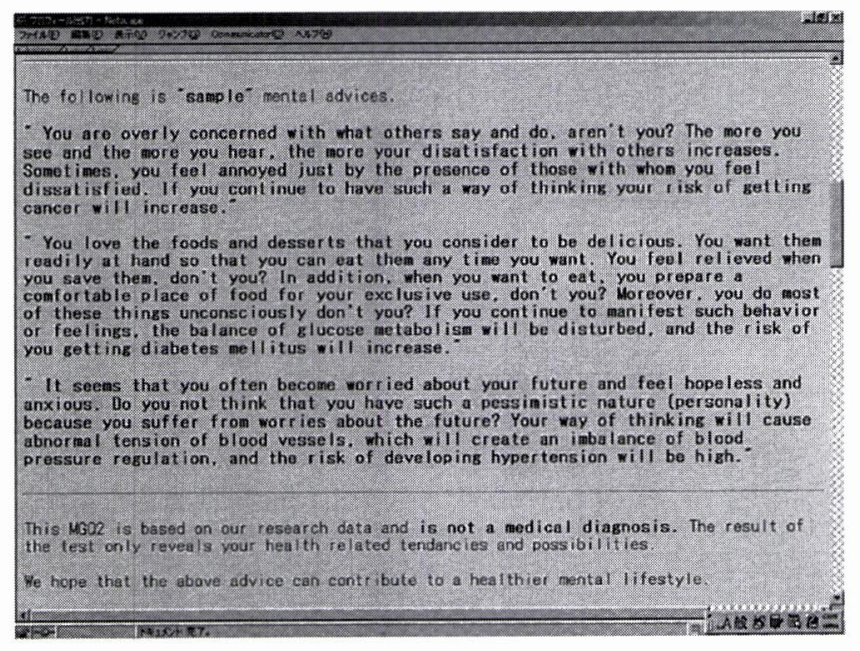

Fig. 3 A sample of the mental advice selected automatically according to a sample profile on the web page.

\section{DISCUSSION}

Health evaluations based on both physical and mental examinations concerning lifestyle-related diseases will be the biggest health science related research subject of the 21 st century. From the beginning of its establishment thirty years ago, the PL Health Control Center concerned itself with both the physical and mental sides of health care. The newest medicine represented by gene diagnosis and diagnostic imaging and so on demonstrates great progress in the physical side of health science during the 20th century. On the other hand, there has not been great progress on the psychological and mental side of health science. This unbalance of progress, favoring only the physical side of human health science and health medicine is not good.

We studied the characteristic personality types or behavior patterns of those with lifestyle-related diseases using studies that were developed mainly thirty years ago. As a result, we recently developed the revised MGQ2, and the mental consulting system using the MGQ2. This system was developed based on the statistical evidence obtained by the analysis of previous personality questionnaires. However, there is no sufficient data yet for an analysis of the revised MGQ2. Another problem is that the MGQ2 is an incomplete questionnaire regarding evidence-based medicine because scales and questions relating to heart disease, apoplexy, and tooth lap disease have not been confirmed by the epidemiological methods. Therefore, our pressing need is the collection of data from the MGQ2 and multiphasic health testing in the health control centers. After the statistical analysis of collected data, we would like to confirm the effectiveness of the personality scales of the MGQ2 on lifestyle-related diseases, to unify the mental consulting system and health risk appraisal using clinical test data, and to make use of this unified comprehensive consulting system which balances health care, both mental and physical.

\section{ACKNOWLEDGEMENTS}

This study was supported by a grant from Patriarch Takahito Miki and the Church of Perfect Liberty, Japan. We thank Rev. Tatsumi Yano and Mr. Paolo Melillo, of the New York Church of 
Perfect Liberty, for kindly correcting the English used in this report.

\section{REFERENCES}

[1] Yasaka, T., and Nakano, K.: Individual physiological variations (individual specific normal values) in blood chemistry and their variance in sex, age and intra-individual variance and inter-individual variance and their applications (in Japanese), JAMHTS, 2(2), 8-19, 1975.

[2] Nakano, K., Atobe, T., Hiraki, Y., and Yasaka, T.: Estimation of subject-specific normal ranges based on some statistical models of an individual's physiological variations, Med. Inform., 6, 195-205, 1981.

[3] Atobe, T., Nakano, Mo., Nakano, K., Nakano, Ma., Kitaike, T., Anan, K., Hiraki, S., Ohno, H., Tamura, M., Kashihara, H., Kawakubo, K., Gunji, A.: Research on disease incidence (4th report) Development of HRA system (in Japanese), Proc. 23rd JAMHTS, 125, 1995.

[4] Friedman, M., and Rosenman, R.H.: Association of specific overt behavior patterns with blood and cardiovascular findings, J.A.M.A., 169,1286-1296, 1959.

[5] Eysenck, H.J.: Personality, cancer and cardiovascular disease: a causal analysis, Person. Individ. Diff., 6(5), 535-556, 1985.

[6] Temoshok, L.: Personality, coping style, emotion, and cancer: Toward an integral model, Cancer Survey, 6(3), 545-567, 1987.

[7] Aoki, S.: Study on the health status of Internet users (in Japanese), Bulletin Gunma University Social Information, 4, 69-90, 1997.

[8] Nakano, K., Yasaka, T., Shinbori, M., and Tamura, M.: Risk factors for gastric cancer: a multivariate analysis of five-years' observation in the PL Health Control System and its applications, Med. Inform., 6, 115-130, 1981.

[9] Nakano, K., Nakao, T., Kashihara, H., Tamura, M., and Kiyoshi- ma, K.: Examination of the personalities of patients with cancer by questionnaire methods (in Japanese), Japanese J. Stress Sciences, 9(2), 70, 1994.

[10] Nakano, K., Aiso, T., Nakao, T., Kiyoshima, K., Kashihara, H., and Tamura, M.: A case-control study of the personalities of patients with gastrointestinal cancers (in Japanese), JMHTS, 24(1), 34-41, 1997.

[11] Nakano, K., Atobe, T., Kiyoshima, K., Kashihara, H., and Tamura, M.: Development of MGQ (mental general questionnaire) [1st Report] factor analysis and its application to health prediction (in Japanese), JMHTS, 23(2), 246, 1996.

[12] Nakano, K., Atobe, T., Aiso, T., Kiyoshima, K., Kashihara, H., and Tamura, M.: Personality and meal behavior of patients with diabetes mellitus (in Japanese), JMHTS, 24(2), 168, 1997.

[13] Nakano, K., Atobe, T., Aiso, T., Kiyoshima, K., Kashihara, H., Ohno, H., and Tamura, M.: Personality of patients with hypertension (in Japanese), Proc. 26th JMHTS, 173, 1998.

[14] Nakano, K., Atobe, T., Kashihara, H., Ohno, H., Kiyoshima, K., and Tamura, M.: Examination of personality as a risk factor of lifestyle-related diseases (in Japanese), Proc. 5th Japanese Behavioral med., 51, 1998.

[15] Nakano, K., Atobe, T., Kashihara, H., Ohno, H., Kiyoshima, K., and Tamura, M.: Development of MGQ (mental general questionnaire) [2nd report] proportional hazard analysis and its application to health prediction (in Japanese), Proc. 27th JMHTS, 94, 1999.

[16] Nakano, K., Matsuoka, M., Iwashita, Y., Ino, Y., Atobe, T., Aiso, T., Haga, H., Suda, M., Kiyoshima, K., Kashihara, H., Ohno, H., and Tamura, M.: Development of MGQ (mental general questionnaire) [3rd report] development of questionnaire for examination of mental background of lifestyle-related diseases (in Japanese), Proc. 28th JMHTS, 234, 2000.

[17] Nakano, K.: Mental Consulting System for Prevention of Life-style Related Diseases, Proc. Int. Health Evaluation and Association (IHEA) 2000 TAIPEI, 2000. 